EPJ Web of Conferences 58, 02009 (2013)

DOI: $10.1051 /$ epjconf/20135802009

(C) Owned by the authors, published by EDP Sciences, 2013

\title{
New covariant Hamilton formalism for field theory
}

\author{
Takayoshi Ootsuka ${ }^{1, a}$
}

${ }^{1}$ Physics Department, Ochanomizu University, 2-1-1 Ootsuka Bunkyo, Tokyo, Japan

\begin{abstract}
A novel covariant Hamilton formalism for field theories is proposed in terms of multi-contact structure which is a higher rank generalization of contact 1-form. This formulation is naturally derived from our previous work, Finsler-Kawaguchi Lagrange formulation. This new covariant Hamilton formulation has a strong covariance so that we can take not only time but also spacetime parameter arbitrarily. In other words, our formulation preliminarily includes the concept of spacetime and we should think of the solution submanifold as our spacetime.
\end{abstract}

\section{Finsler-Kawaguchi Lagrange formalism}

We usually start Lagrange formalism from $(Q, L)$ where $Q$ is a configuration manifold and $L$ is a Lagrangian of a system, and in general $(Q, L)$ cannot have a (metric) geometry. So the Euler-Lagrange equation and variational principle depend on parameterisation of the dynamical curve in $Q$. However, it is known that there is a geometric formulation of Lagrange systems using Finsler geometry [13]. Instead of $Q$, we should think the extended configuration manifold $M=\mathbb{R} \times Q$ and we can define a structure called a Finsler function (Finsler metric, Finsler 1-form) $F$ from the Lagrangian $L\left(q^{i}, \dot{q}^{i}, t\right),(i=1,2, \ldots, n)$ by,

$$
F\left(x^{0}, x^{i}, d x^{0}, d x^{i}\right)=L\left(x^{i}, \frac{d x^{i}}{d x^{0}}, x^{0}\right) d x^{0} .
$$

It is important that Finsler 1 -form $F(x, d x)$ which is a function of $x^{0}, x^{i}, d x^{0}, d x^{i}$ satisfies the following homogeneity condition,

$$
F\left(x^{\mu}, \lambda d x^{\mu}\right)=\lambda F\left(x^{\mu}, d x^{\mu}\right), \quad \lambda>0 .
$$

In application to physics, the other assumptions of $F(x, y) ; 1) \operatorname{det}\left(g_{\mu v}\right) \neq 0, g_{\mu \nu}(x, y)=\frac{1}{2} \frac{\partial^{2} F^{2}}{\partial y^{\mu} \partial y^{v}}$ 2) $F(x, y) \geq 0$ in mathematical context is not needed, because constraint (gauge) systems define degenerate Finsler metrics and if necessarily using properly large positive constant $E$ we can define $\tilde{F}=F+E d x^{0}$ which is positive without change of their dynamics. By this homogeneity condition Finsler 1-form $F(x, d x)$ can define a geometric norm for a tangent vector $v \in T_{p} M$,

$$
\|\boldsymbol{v}\|=F\left(x^{\mu}(p), d x^{\mu}(\boldsymbol{v})\right),
$$

\footnotetext{
ae-mail: ootsuka@cosmos.phys.ocha.ac.jp
}

This is an Open Access article distributed under the terms of the Creative Commons Attribution License 2.0, which permits unrestricted use, distribution, and reproduction in any medium, provided the original work is properly cited. 
and for oriented curve $c \subset M$ a geometric length,

$$
\mathcal{A}[\boldsymbol{c}]=\int_{\boldsymbol{c}} F(x, d x)=\int_{\tau_{0}}^{\tau_{1}} F\left(x(\tau), \frac{d x(\tau)}{d \tau}\right) d \tau,
$$

which is independent of parameterisation $\tau$ for curve $\boldsymbol{c}$. In this formulation, an oriented curve $\boldsymbol{c}$ represents an orbital of a system and Finsler length $\mathcal{A}[\boldsymbol{c}]$ corresponds to the action of the system, so the variational principle and Euler-Lagrange equation become geometric and parameterisation invariant (covariant). Using this covariant formulation, we can take parameter for convenience of calculation, and we think that there is theoretical deep importance. When we think a path integral quantisation [4], we showed that Finsler structure define a Feynman measure geometrically and reparameterisation transforms a path integral non-perterbatively.

In the same way of finite dimensional Lagrange systems, we can reformulate field Lagrange systems to covariant and geometric one [5,6]. An extended field configuration manifold which is spacetime and field configuration space and Kawaguchi function (areal metric, Kawaguchi metric) defined from field Lagrangian becomes a Kawaguchi manifold which is a higher dimensional generalisation of Finsler manifold.

For an easy understanding, we will think of a scalar field theory in 2-dimensional spacetime, whose Lagrangian is $L=\frac{1}{2} u_{t}^{2}-\frac{1}{2} u_{x}^{2}-V(u)$. Extended field configuration space becomes $M=\mathbb{R}^{3}=$ $\left\{\left(x^{0}, x^{1}, x^{2}\right)\right\}=\{(t, x, u)\}$ and Kawaguchi metric (Kawaguchi 2-form) $K(x, d x)$ is defined by

$$
K\left(x^{0}, x^{1}, x^{2}, d x^{01}, d x^{21}, d x^{02}\right)=\frac{\left(d x^{21}\right)^{2}-\left(d x^{02}\right)^{2}}{2 d x^{01}}-V\left(x^{2}\right) d x^{01},
$$

where we use the notation like $d x^{21}=d x^{2} \wedge d x^{1}$. This Kawaguchi 2-form also satisfies the following homogeneity condition,

$$
K\left(x^{\mu}, \lambda d x^{\mu \nu}\right)=\lambda K\left(x^{\mu}, d x^{\mu \nu}\right), \quad \lambda>0 .
$$

By this property, Kawaguchi 2-form $K$ can define an geometric area $\mathcal{A}[\sigma]=\int_{\sigma} K(x, d x)$ for an oriented surface $\sigma \subset M$ independently of parameterisation. Same way of Finsler-Lagrange formulation, oriented surfaces $\sigma$ represent field configuration and dynamics and Kawaguchi area $\mathcal{A}[\sigma]$ corresponds to a geometric action of field Lagrange system. Using this formulation, we can easily define an energy-momentum of gravity from Nöther theorem and reformulate field path integral.

However, while these Finsler-Kawaguchi formulation of Lagrange systems are suitable for considering symmetries or constructing new theories, they are weak in discovering new symmetries or solutions. Therefore, we should construct a Hamiltonian version of these new Lagrange formalism.

\section{Covariant Hamilton formalism for particles}

In the case of Finser-Lagrange formulation of finite dimensional Lagrange systems, a covariant Hamilton formulation [7] can be easily derived.

When we define covariant conjugate momentums by $p_{\mu}=\frac{\partial F}{\partial d x^{\mu}},(\mu=0,1, \cdots, n)$, the following formula can be derived from the homogeneity condition of $F$ and Euler's formula,

$$
F(x, d x)=\frac{\partial F}{\partial d x^{\mu}} d x^{\mu}=p_{\mu} d x^{\mu}
$$

Furthermore, since $p_{\mu}$ satisfy 0 -th degree homogeneity condition,

$$
p_{\mu}\left(x^{\nu}, \lambda d x^{\nu}\right)=p_{\mu}\left(x^{\nu}, d x^{\nu}\right), \quad \lambda>0
$$


using Euler's formula, following condition is derived,

$$
\frac{\partial p_{\mu}}{\partial d x^{\nu}} d x^{\nu}=0 \Rightarrow \operatorname{det}\left(\frac{\partial p_{\mu}}{\partial d x^{v}}\right)=0 .
$$

So we should think more than one constraint $G\left(x^{\mu}, p_{\mu}\right)=0$ for Legendre transformation $\left(x^{\mu}, d x^{\mu}\right)$ to $\left(x^{\mu}, p_{\mu}\right)$.

Let us think there is one such constraint $G=0$ in the case of potential systems. We define a $2 n+1$-dimensional extended phase manifold $\Sigma \subset T^{*} M^{n+1}$ and 1-form $\theta$ on $\Sigma$,

$$
\Sigma:=\left\{\left(x^{\mu}, p_{\mu}\right) \in T^{*} M ; G(x, p)=0\right\}, \quad \theta:=\left.p_{\mu} d x^{\mu}\right|_{G=0} .
$$

Then we can prove that $\theta$ becomes a contact 1 -form, that is; $\theta \wedge(d \theta)^{n} \neq 0$. Therefore $(\Sigma, \theta)$ becomes a contact manifold and so this covariant Hamilton formalism is a contact geometry. In this formulation the generator of dynamics is given by the vector field $X$ satisfying the following equation (Cartan equation),

$$
\iota_{X} d \theta=0 .
$$

If a vector field $X$ satisfy (11), $X^{\prime}=f X$ also satisfies (11) where $f$ is an arbitrary positive function of $\Sigma$. So $X$ has an ambiguity and the ambiguity reflects reparameterisation invariance (covariance) of this formulation. Using this covariance we can take time parameter for convenience of calculation. If we take the time parameter $s$ which is a function of $\Sigma$, the ambiguity of $X$ is deleted from the time gauge condition; $\langle d s, X\rangle=X(s)=1$. For a function (physical quantity) $\mathcal{F}$ on $\Sigma$

$$
\frac{d \mathcal{F}}{d s}=X(\mathcal{F})
$$

becomes evolution equation (Hamilton equation) about time parameter $s$. If the contact 1 -form $\theta$ can be decomposable for this parameter s such as $\theta=p-\mathcal{H} d s$, then the $\theta$ corresponds to Poincaré-Cartan form. The 1-form $\theta$ is also called Hilbert form in variational calculus or Finsler geometry [8].

\section{New covariant Hamilton formalism for fields}

Unlike the previous section we will define a new covariant field Hamilton formalism [9] in an abstract way. We think a manifold $\Sigma$ and $(n+1)$-form $\Theta$ on $\Sigma$ which satisfies

$$
\Theta \wedge(d \Theta)^{k}=\Theta \wedge d \Theta \wedge \cdots \wedge \Theta \neq 0,
$$

where $\Theta \wedge(d \Theta)^{k}$ is a top-form on $\Sigma$. Therefore the dimension of $\Sigma$ is $(k+1) n+2 k+1$. We will call $\Theta$ "multi-contact form" and $(\Sigma, \Theta)$ "multi-contact manifold" which is not the same multi-contact structure called in [10]. Field configuration and dynamics, namely a $(n+1)$-dimensional submanifold $\tilde{\sigma} \subset \Sigma$, is determined by $(n+1)$-vector field $X$ given by the following Cartan equation for field,

$$
\iota_{X} d \Theta=0 .
$$

Let us consider this formulation for concrete example, a scalar field theory on 2-dimensional spacetime similarly as in the previous section. As in the finite dimensional Lagrange case, we can introduce a covariant Hamilton formulation for field naturally by Kawaguchi-Lagrange formulation. We define covariant conjugate momentums by $\pi_{\mu \nu}=\frac{\partial K}{\partial d x^{\mu \nu}},(\mu, v=0,1,2)$. If $K(x, d x)$ is defined by (5), they are

$$
\pi_{21}=\frac{d x^{21}}{d x^{01}}, \quad \pi_{02}=\frac{d x^{02}}{d x^{01}}, \quad \pi_{01}=-\frac{1}{2}\left(\frac{d x^{21}}{d x^{01}}\right)^{2}+\frac{1}{2}\left(\frac{d x^{02}}{d x^{01}}\right)^{2}-V .
$$


Then there is one constraint,

$$
G(x, \pi)=\pi_{01}+\frac{1}{2}\left(\pi_{21}\right)^{2}-\frac{1}{2}\left(\pi_{02}\right)^{2}+V=0 .
$$

Therefore, we define an extended field phase space $\Sigma \subset \Lambda^{2} T^{*} M$ and 2-form $\Theta^{\prime}$ on $\Sigma$,

$$
\Sigma:=\left\{(x, \pi) \in \Lambda^{2} T^{*} M ; G(x, \pi)=0\right\}=\mathbb{R}^{5}, \quad \Theta^{\prime}:=\left.\frac{1}{2} \pi_{\mu \nu} d x^{\mu \nu}\right|_{G=0} .
$$

If we take a coordinate $\left(x^{0}, x^{1}, x^{2}, \pi_{21}, \pi_{02}\right)$ for $\Sigma$, the 2 -form $\Theta^{\prime}$ becomes

$$
\Theta^{\prime}=\pi_{21} d x^{21}+\pi_{02} d x^{02}+\left\{-\frac{1}{2}\left(\pi_{21}\right)^{2}+\frac{1}{2}\left(\pi_{02}\right)^{2}-V\right\} d x^{01}
$$

Since $\Theta^{\prime}$ satisfies $\Theta^{\prime} \wedge d \Theta^{\prime}=0, \Theta^{\prime}$ is not a multi-contact 2-form, but we can fix it by adding exact 2-form such as $\Theta:=\Theta^{\prime}+d \pi_{21} \wedge d x^{0}+d \pi_{02} \wedge d x^{1}$. Then we can get a multi-contact manifold $(\Sigma, \Theta)$. From this multi-contact 2-form $\Theta$ and Cartan equation (14), we get

$$
\begin{array}{r}
X=v^{01}\left(\frac{\partial}{\partial x^{0}} \wedge \frac{\partial}{\partial x^{1}}-\pi_{02} \frac{\partial}{\partial x^{0}} \wedge \frac{\partial}{\partial x^{2}}+\pi_{21} \frac{\partial}{\partial x^{2}} \wedge \frac{\partial}{\partial x^{1}}-V^{\prime} \frac{\partial}{\partial \pi_{21}} \wedge \frac{\partial}{\partial x^{1}}-\pi_{02} V^{\prime} \frac{\partial}{\partial \pi_{21}} \wedge \frac{\partial}{\partial x^{2}}\right) \\
+v_{21}{ }^{0}\left(\frac{\partial}{\partial \pi_{21}} \wedge \frac{\partial}{\partial x^{0}}+\pi_{21} \frac{\partial}{\partial \pi_{21}} \wedge \frac{\partial}{\partial x^{2}}\right)+v_{02}{ }^{1}\left(\frac{\partial}{\partial \pi_{02}} \wedge \frac{\partial}{\partial x^{1}}-\pi_{02} \frac{\partial}{\partial \pi_{02}} \wedge \frac{\partial}{\partial x^{2}}\right) \\
+v_{02}{ }^{0}\left(\frac{\partial}{\partial \pi_{02}} \wedge \frac{\partial}{\partial x^{0}}+\frac{\partial}{\partial \pi_{21}} \wedge \frac{\partial}{\partial x^{1}}-\pi_{02} \frac{\partial}{\partial \pi_{21}} \wedge \frac{\partial}{\partial x^{2}}+\pi_{21} \frac{\partial}{\partial \pi_{02}} \wedge \frac{\partial}{\partial x^{2}}\right)+v_{21,02} \frac{\partial}{\partial \pi_{21}} \wedge \frac{\partial}{\partial \pi_{02}}
\end{array}
$$

where $v^{01}, v_{21}{ }^{0}, v_{02}{ }^{0}, v_{02}{ }^{1}, v_{21,02}$ are arbitrary (gauge) functions. The integrable submanifold defined by this 2-vector field becomes field configuration and dynamics.

Finally we will re-express this by evolution form (covariant Hamilton equation). Since our formalism is covariant, we can adopt arbitrary spacetime parameter, but for easily understanding we take parameters $s^{0}=x^{0}, s^{1}=x^{1}$. In this spacetime parameterisation, $v^{01}=1$ is derived from the spacetime gauge condition; $\left\langle d s^{0} \wedge d s^{1}, X\right\rangle=v^{01}=1$. Then the Hamilton equation for field becomes

$$
\frac{d \mathcal{F}}{d x^{01}}=\iota_{X} d \mathcal{F} \text {. }
$$

In the above equation $\mathcal{F}$ represents physical quantity and mathematically becomes a 1 -form in this 2-dimensional field theory example. The LHS of the equation means the pull-back of $\mathcal{F}$ to parameter space $\left\{\left(x^{0}, x^{1}\right)\right\}$ and doing exterior derivative $d \mathcal{F}=\left(\frac{\partial \mathcal{F}_{1}}{\partial x^{0}}-\frac{\partial \mathcal{F}_{0}}{\partial x^{1}}\right) d x^{01}$ and then divided by $d x^{01}$.

Using this evolutionary equation (20), let us check the validity of this formalism. If we take $\mathcal{F}$ as $\mathcal{F}=x^{2} d x^{1}$,

$$
\frac{d \mathcal{F}}{d x^{01}}=\frac{d x^{2} \wedge d x^{1}}{d x^{01}}=\frac{\partial_{0} x^{2} d x^{01}}{d x^{01}}=\partial_{0} x^{2}=\partial_{0} u=\pi_{21}=\iota_{X} d x^{21}=\iota_{X} d \mathcal{F} .
$$

Same way we take $\mathcal{F}_{1}=-x^{2} d x^{0}, \mathcal{F}_{2}=\pi_{21} d x^{1}, \mathcal{F}_{3}=\pi_{02} d x^{0}$,

$$
\begin{aligned}
& \frac{d \mathcal{F}_{1}}{d x^{01}}=\frac{d x^{0} \wedge d x^{2}}{d x^{01}}=\frac{\partial_{1} x^{2} d x^{01}}{d x^{01}}=\partial_{1} x^{2}=\partial_{1} u=-\pi_{02}=\iota_{X} d x^{02}=\iota_{X} d \mathcal{F}_{1}, \\
& \frac{d \mathcal{F}_{2}}{d x^{01}}=\frac{d \pi_{21} \wedge d x^{1}}{d x^{01}}=\frac{\partial_{0} \pi_{21} d x^{01}}{d x^{01}}=\partial_{0} \pi_{21}=-V^{\prime}+v_{02}{ }^{0}=\iota_{X} d \pi_{21} \wedge d x^{1}=\iota_{X} d \mathcal{F}_{2}, \\
& \frac{d \mathcal{F}_{3}}{d x^{01}}=\frac{d \pi_{02} \wedge d x^{0}}{d x^{01}}=-\frac{\partial_{1} \pi_{02} d x^{01}}{d x^{01}}=-\partial_{1} \pi_{02}=v_{02}{ }^{0}=\iota_{X} d \pi_{02} \wedge d x^{0}=\iota_{X} d \mathcal{F}_{3} .
\end{aligned}
$$

Then we can check the equation of motion of the scalar field,

$$
\partial_{0} \pi_{21}+\partial_{1} \pi_{02}+V^{\prime}=0 \quad \Leftrightarrow \quad \partial_{0}^{2} u-\partial_{1}^{2} u+V^{\prime}=0 .
$$




\section{References}

[1] C. Lanczos, The Variational Principles of Mechanics (Dover, New York, USA,1986)

[2] Y. Suzuki, Journal of the College of Arts and Sciences, Chiba university, 2, 1, 12-16 (1956)

[3] H. Goldstein, C.P. Poole, J.F. Safko, Classical Mechanics, (Pearson Education, London, UK, 2001)

[4] T. Ootsuka, E. Tanaka, Physics Letters A 374, 1917-1921 (2010)

[5] T. Ootsuka, arXiv:1206.6040v1 (2012)

[6] E. Tanaka, "General Relativity by Kawaguchi geometry", in this Proceedings (2012)

[7] V. Aldaya, J. A. De Azcárraga, Riv. Nuovo Cimento, 3, 10, 1-66 (1980)

[8] S.S. Chern, W.H. Chen, K.S. Lam, Lectures On Differential Geometry, (World Scientific, Singapore, 1999)

[9] T. Ootsuka, "New covariant Hamilton formulation for field theories", in preparation

[10] R. Bryant, P. Griffiths, D. Grossman, Exterior Differential Systems and Euler-Lagrange Partial Differential Equations, (Chicago Lectures in Mathematics, Chicago, USA, 2003) 
